\title{
SÍNTESE E CARACTERIZAÇÃO DE MATERIAL HÍBRIDO TIPO HIDROTALCITA-HIDROXIAPATITA
}

\author{
H. F. F. BRASIL ${ }^{1}$, P. M. PEREIRA ${ }^{2}$, J. A. M. CORREA $^{2}$, E. M. S. RODRIGUES ${ }^{3}$ \\ ${ }^{1}$ Universidade Federal do Pará, Faculdade de Engenharia Química \\ ${ }^{2}$ Universidade Federal do Pará, Programa de Pós Graduação em Geologia e Geoquímica \\ ${ }^{3}$ Universidade Federal Pará, Faculdade de Química \\ E-mail para contato: henriquefernandes@ outlook.com
}

\begin{abstract}
RESUMO - A demanda por novos materiais com aplicações diversificadas aumenta com o crescimento industrial e com o desenvolvimento de novas tecnologias. Materiais como hidrotalcita e hidroxiapatita são conhecidos por seus empregos como adsorventes no tratamento de efluentes, como catalisadores e suporte para catalisadores, entre outros. Esse trabalho objetiva a síntese e caracterização de um material hibrido tipo hidrotalcitahidroxiapatita (HT-HAp) para futuros testes de adsorção e catálise. A síntese foi realizada pelo método de co-precipitação e pH variando entre 10 e 11, homogeneização ultrassônica e tratamento hidrotérmico a $60^{\circ} \mathrm{C}$. A caracterização foi realizada pelas técnicas de DRX, FTIR e MEV-EDS. Os resultados obtidos confirmaram a cristalização do material HTHAp, indicando assim a eficiência da rota de síntese.
\end{abstract}

\section{INTRODUÇÃO}

O uso de novos materiais bifuncionais, que apresentem tanto características ácidas quanto básicas, são cada vez mais desejáveis devido a uma aplicabilidade mais ampla nos processos catalíticos industriais. Nesse sentido, a hidrotalcita (HT), que possui caráter básico foi combinada com a hidroxiapatita (HAp), de funcionalidade ácida a fim de produzir um material híbrido tipo HTHAp. Os materiais de partida são hidróxidos sólidos bem conhecidos com propriedades de troca iônica repertoriada pela literatura (Rivera et al., 2009).

\subsection{Hidrotalcita (HT)}

A fórmula geral que descreve a composição química da HT é indicada por:

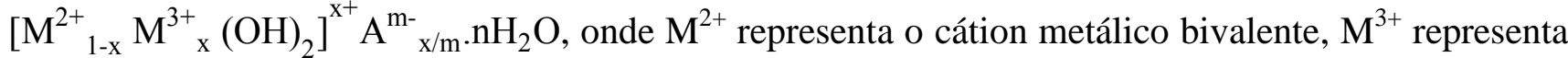
o cátion metálico trivalente, $\mathrm{A}^{\mathrm{m}-}$ é o ânion de compensação e x é a razão molar $\mathrm{M}^{3+} / \mathrm{M}^{2+}+\mathrm{M}^{3+}$ (Cavani et al., 1991).

As HTs, também denominadas de argilas aniônicas e hidróxidos duplos lamelares (HDLs), apresentam folhas octaédricas exibindo combinações de um ou mais metais bivalentes e trivalentes e um espaço interlamelar contendo ânions e moléculas de água (Crepaldi; Valim, 1998) (Figura 1). Apresentam uma grande variedade de aplicações, dependendo de sua composição, cristalinidade, 
estabilidade térmica e outras propriedades físico-químicas. Entre as aplicações mais usuais pode-se citar o uso como catalisadores, suportes catalíticos, adsorventes e drug deliveries (Cardoso, 2002).

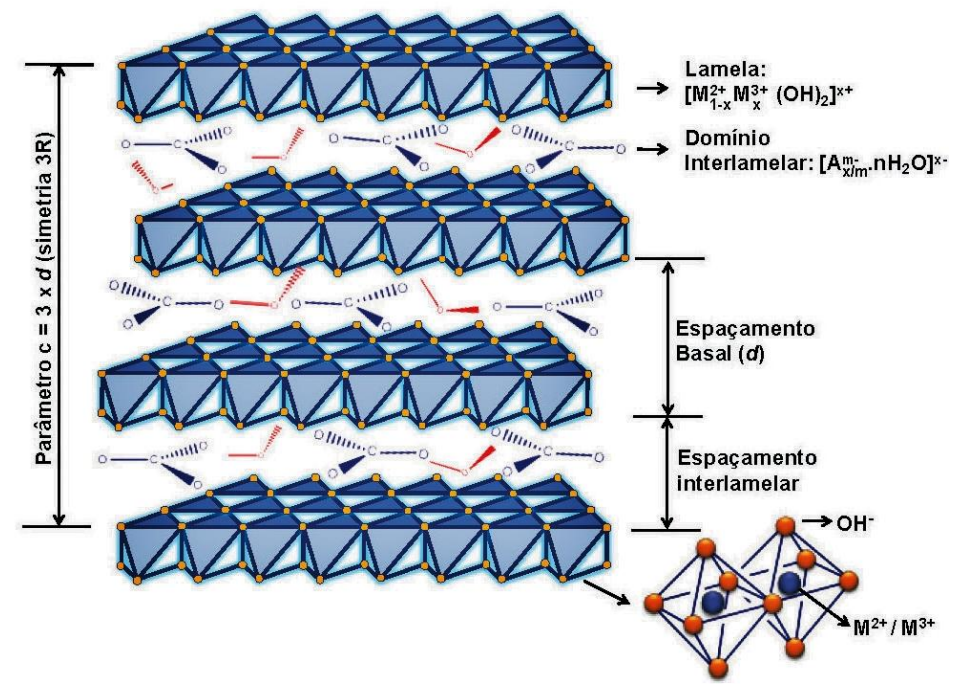

Figura 1 - Representação esquemática da estrutura dos HDL (Cunha et al., 2010).

As HTs têm sido bastante utilizadas como precursoras para outros materiais ativos de uso catalítico, favorecidos pela presença de ânions altamente móveis e a basicidade das camadas superficiais. Outros usos incluem troca iônica, adsorção aniônica, e estabilização molecular. A facilidade de controlar a composição das argilas aniônicas e a possibilidade de aumentar a área superficial ativa e o volume interlamelar juntamente com a escolha apropriada de ânions intercalados permite um ótimo ajuste de suas propriedades, possibilitando sintetizar um material de acordo com a finalidade que se deseja (Trave et al., 2002).

\subsection{Hidroxiapatita (HAp)}

A HAp apresenta formula química $\left[\mathrm{Ca}_{10}\left(\mathrm{PO}_{4}\right)_{6}(\mathrm{OH})_{2}\right]$, com razão $\mathrm{Ca} / \mathrm{P}=1.67$, sendo o mineral mais estável e o menos solúvel dentre os fosfatos de cálcio (Fulmer; Martin; Brown, 1992). Cristaliza no sistema hexagonal com grupo espacial $\mathrm{P}_{3 / \mathrm{m}}$, onde $\mathrm{P}$ indica um sistema hexagonal primitivo (Guastaldi; Aparecida, 2010) (Figura 2).

A HAp é um constituinte mineral do osso natural representando de 30 a $70 \%$ da massa dos ossos e dentes. A HAp sintética possui propriedades de biocompatibilidade e osteointegração o que a torna substituta do osso humano em implantes e próteses (Eanes, 1980). 


\section{9 a 22 de outubro de 2014 \\ Florianópolis/SC}

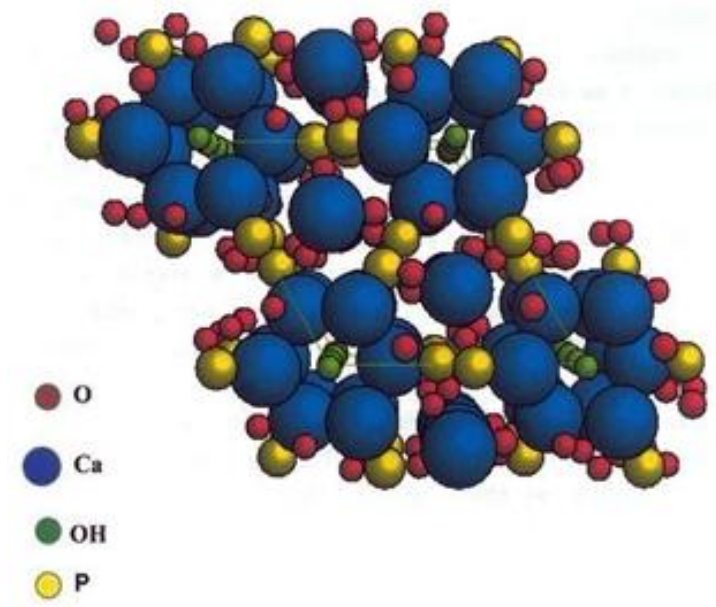

Figura 2 - Representação esquemática da estrutura da hidroxiapatita (Kay; Young, 1964 apud Mavropoulos, 1999).

A estrutura da HAp permite substituições catiônicas e aniônicas isomorfas com grande facilidade, sendo aplicada como adsorvente em cromatografia líquida (Akazawa; Kobayashi, 1996), catalisador na decomposição de compostos orgânicos clorados poluentes provenientes da indústria metalúrgica e da incineração do lixo industrial, e na remoção de metais pesados, não somente de águas e solos contaminados, como também de dejetos industriais (Ma et al., 1993).

\section{MATERIAIS E MÉTODOS}

A síntese do material foi realizada por meio do método de co-precipitação e $\mathrm{pH}$ variando entre 10 e 11. Os compostos HT e HAp foram sintetizados separadamente e homogeneizados em banho ultrassônico.

A síntese da HT envolveu duas etapas: preparo de uma solução de $\mathrm{NaOH} 3 \mathrm{M}$ para dissolução do alumínio metálico e preparo de uma solução de $\mathrm{Mg}\left(\mathrm{NO}_{3}\right)_{2} \cdot 6 \mathrm{H}_{2} \mathrm{O} 0,24 \mathrm{M}$, obtidas segundo a estequiometria da HT, a partir da metodologia proposta por Reichle (1985).

Na síntese da HAp foram preparadas duas soluções: uma de $\mathrm{H}_{3} \mathrm{PO}_{4} 0,3 \mathrm{M}$ e outra de $\mathrm{Ca}(\mathrm{OH})_{2}$ 0,5M. Em seguida, foi realizado o gotejamento da solução de $\mathrm{H}_{3} \mathrm{PO}_{4}$ na $\mathrm{HAp}$ e da solução $\mathrm{NaOH}-\mathrm{Al}$ na HT até o pH igual a 10. O fluxograma de síntese do material tipo HT-HAp é indicado na Figura 3. A reação ocorreu à temperatura ambiente, com fluxo de gotejamento igual a $1 \mathrm{~mL} / \mathrm{min}$ e agitação magnética.

As soluções obtidas nos processos anteriores foram mantidas separadas em banho ultrassônico por $30 \mathrm{~min}$. Após esse período, foram misturadas e permaneceram em banho ultrassônico por $2 \mathrm{~h}$. $\mathrm{O}$ precipitado obtido foi submetido a banho hidrotérmico por $24 \mathrm{~h} / 80^{\circ} \mathrm{C}$, em seguida foi lavado até atingir $\mathrm{pH}=7$, filtrado e seco em estufa por $24 \mathrm{~h} / 80^{\circ} \mathrm{C}$. 


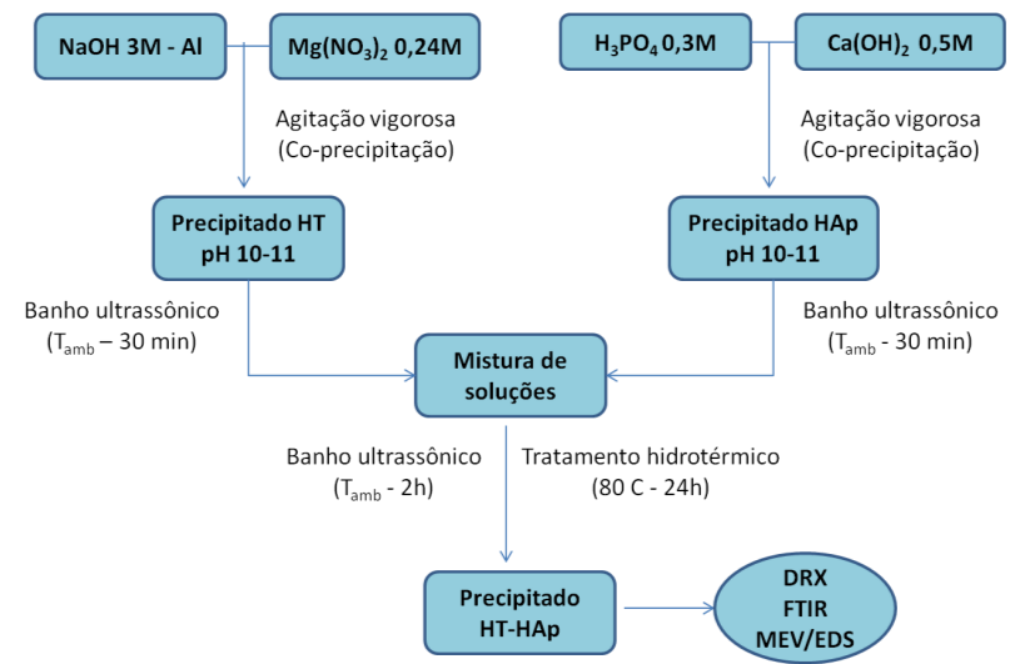

Figura 3. Fluxograma de síntese do material hibrido tipo HT-HAp.

\subsection{Caracterização do material}

As amostras sintetizadas foram caracterizadas utilizando-se as técnicas de Difração de Raios-X (DRX), Espectroscopia na Região do Infravermelho (FTIR) e Microscopia Eletrônica de Varredura (MEV/EDS). As condições de análise foram as seguintes:

Difração de Raios-X: PANalytical XPERT-PRO PW 3050, Cu $K \alpha(\lambda=1,5406 \AA$ A), 20s, $30 \mathrm{~mA}$, $40 \mathrm{kV}$, software X'PERT HighScore, banco de dados International Center for Diffraction Data (ICDD).

Espectroscopia na Região do Infravermelho: SHIMADZU, IRPrestige21, 400 a $4000 \mathrm{~cm}^{-1}$, resolução de $4 \mathrm{~cm}^{-1}$ e 16 varreduras.

Microscopia Eletrônica de Varredura: Zeiss - LEO 1430, metalização em Au, recobrimento de 2 min., $90 \mu \mathrm{A}, 10 \mathrm{Kv}, 15 \mathrm{~mm}$.

\section{RESULTADOS}

Os resultados das caracterizações físico-químicas do material sintetizado, avaliando os aspectos texturais, morfológicos e químicos são apresentados a seguir.

\subsection{Difração de Raios-X}

No difratograma foram identificados os planos de maior intensidade das fases HT e HAp. O material HT-HAp apresentou reflexões características da HT [(003), (006), (012) e (018)] e da HAp [(211), (300) (002), (213) e (202)] indexados de acordo com as fichas PDF 01-089-0460 (ICDD, 1996) e 01-072-1243 (ICDD, 1997), respectivamente (Figura 4). 


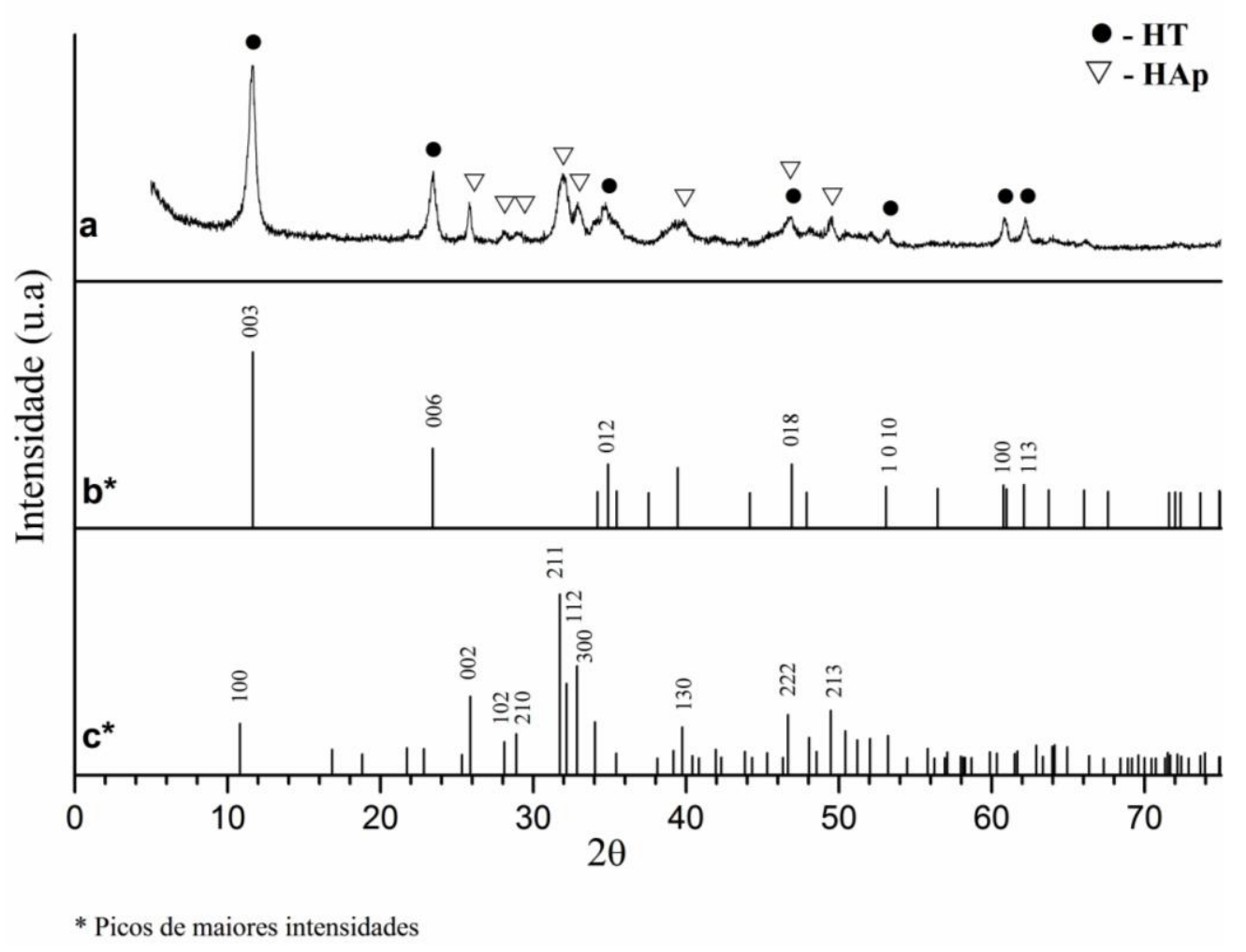

Figura 4 - Difratogramas do material HT-HAp (a), HT de referência (b) e HAp de referência (c).

A cristalização das fases HT e HAp indicou ligeiras alterações nas distâncias interplanares e ausência do pico (112) na fase HAp. Os picos da HT são mais intensos e bem definidos em relação aos da HAp, indicando que provavelmente que essa fase predomina no material híbrido. Os parâmetros de rede do material HT-HAp são apresentados na Tabela 1.

Tabela 1 - Parâmetros de rede do material HT-HAp, comparados com as fichas de referência

\begin{tabular}{c|cc|cc}
\hline & HT PDF 01-089-0460 & HT-HAp & HAp PDF 01-072-1243 & HT-HAp \\
\hline a=b $(\AA)$ & 3,046 & $3,00 \pm 0,04$ & 9,432 & $9,46 \pm 0,04$ \\
$\mathbf{c}(\AA)$ & 22,772 & $22,8 \pm 0,82$ & 6,881 & $6,91 \pm 0,02$ \\
$\mathbf{V}\left(\AA^{3}\right)$ & 182,97 & $178,2 \pm 7,9$ & 530,123 & $534,8 \pm 4,6$ \\
\hline
\end{tabular}

Os valores obtidos são concordantes com os do banco de dados consultado, comprovando assim a cristalinidade do material. Os parâmetros da fase HT apresentam um desvio aproximadamente nulo. Acredita-se que substituições de cátions e ânions na estrutura da HAp resultem em alterações no parâmetro de rede, tais como o aumento no volume que pode ser atribuído à deformações decorrentes da inserção aniônica do íon carbonato $\left(\mathrm{CO}_{3}{ }^{2-}\right)$. 


\subsection{Espectroscopia na Região do Infravermelho (FTIR)}

A análise do espectro FTIR indicou a presença dos grupos inorgânicos hidroxila $\left(\mathrm{OH}^{-}\right)$, carbonato $\left(\mathrm{CO}_{3}{ }^{2-}\right)$, fosfato $\left(\mathrm{PO}_{4}{ }^{3-}\right)$ e moléculas de água $\left(\mathrm{H}_{2} \mathrm{O}\right)$. A presença de bandas de adsorção em torno de $3.500 \mathrm{~cm}^{-1}$ foi atribuída às vibrações de estiramento $\mathrm{O}-\mathrm{H}$ das moléculas de água adsorvidas e aos grupos $\left(\mathrm{OH}^{-}\right)$da HAp. Uma banda referente à deformação angular da água foi identificada em torno de $1600 \mathrm{~cm}^{-1}$ (Figura 5).

O ânion $\mathrm{CO}_{3}{ }^{2-}$ da $\mathrm{HT}$ foi caracterizado por bandas de absorções ativas no FTIR as quais ocorreram em torno de $780 \mathrm{~cm}^{-1}$. A presença de uma banda na região de $1360 \mathrm{~cm}^{-1}$ foi atribuída à redução da simetria desse ânion (sítio de simetria $\mathrm{C} 2 \mathrm{v}$ ) e à desordem natural no espaço interlamelar (Bastiani, 2001 apud Conceição et al., 2007).

As bandas entre 500 e $900 \mathrm{~cm}^{-1}$ indicaram vibrações metal-oxigênio (M-O), portanto, correspondentes às vibrações das ligações $\mathrm{Al}-\mathrm{O}$ e $\mathrm{Mg}-\mathrm{O}$ na HT. As bandas intensas em torno de 1090 $\mathrm{cm}^{-1}$ e em $1030 \mathrm{~cm}^{-1}$ são típicas do modo de estiramento antissimétrico dos ânions $\mathrm{PO}_{4}{ }^{3-}$ na $\mathrm{HAp}$.

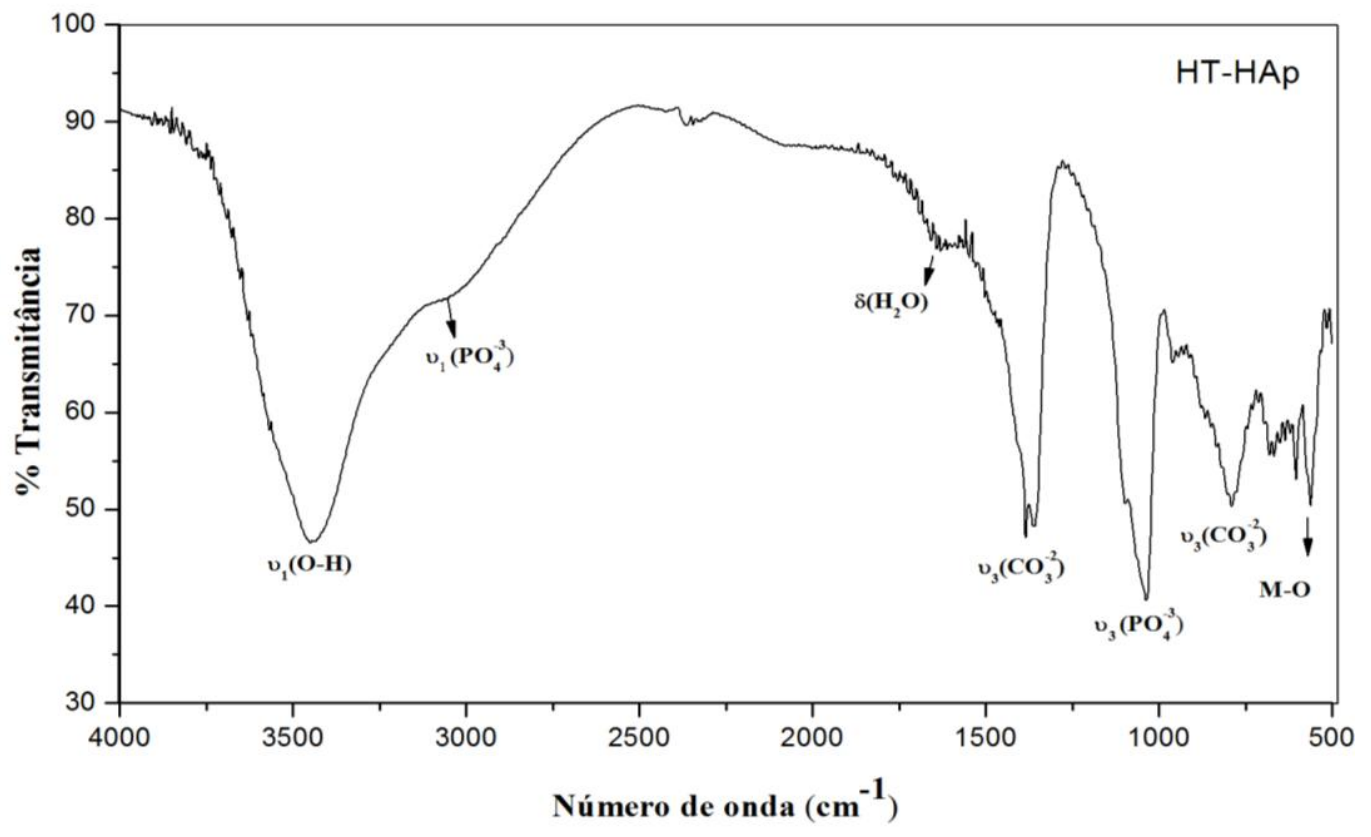

Figura 5 - Espectro FTIR do material HT-HAp.

\subsection{Microscopia Eletrônica de Varredura (MEV/EDS)}

Os dados MEV/EDS para o material sintetizado evidenciaram a presença de aglomerados de tamanhos micrométricos (2 a $10 \mu \mathrm{m})$ (Figura 6). A análise química qualitativa (EDS) indicou a presença de $\mathrm{Ca}, \mathrm{P}, \mathrm{Mg}, \mathrm{Al}$ e $\mathrm{O}$, apresentando as razões $\mathrm{Ca} / \mathrm{P}=1,36$ e $\mathrm{Mg} / \mathrm{Al}=4,5$ (Tabela 2). 


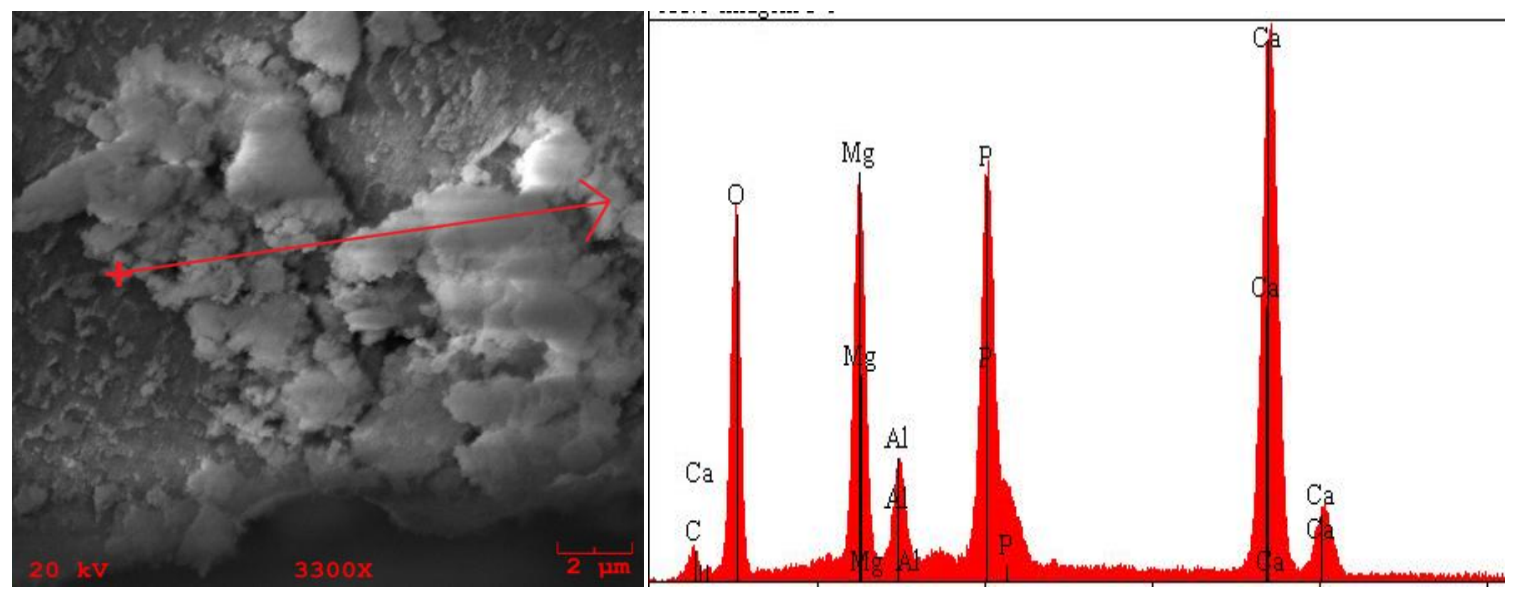

Figura 6-Micrografia do material HT-HAp.

Tabela 2 - Composição química estimada do material no ponto 1

\begin{tabular}{ccccccc}
\hline Elemento & $\mathrm{C}$ & $\mathrm{O}$ & $\mathrm{Mg}$ & $\mathrm{Al}$ & $\mathrm{P}$ & $\mathrm{Ca}$ \\
\hline Concentracao (\% p/p) & 2,31 & 48,27 & 11,82 & 2,91 & 12,55 & 22,13 \\
Concentracao (\%at) & 4,04 & 63,36 & 10,22 & 2,27 & 8,51 & 11,60 \\
\hline
\end{tabular}

As fórmulas químicas dos materiais $\mathrm{HT} \quad\left[\mathrm{Mg}_{6} \mathrm{Al}_{2}(\mathrm{OH})_{16} \mathrm{CO}_{3} \cdot 4 \mathrm{H}_{2} \mathrm{O}\right]$ e $\mathrm{HAp}$ $\left[\mathrm{Ca}_{10}\left(\mathrm{PO}_{4}\right)_{6}(\mathrm{OH})_{2}\right]$ fornecem as razões estequiométricas $\mathrm{Mg} / \mathrm{Al}=3$ e $\mathrm{Ca} / \mathrm{P}=1,67$. A razão $\mathrm{Mg} / \mathrm{Al}$ do material híbrido está dentro do valor esperado para a composição da HT que varia entre 1 e 8 (Cardoso, 2002). A razão Ca/P está abaixo do valor de referência indicando possíveis mudanças na estrutura da HAp e variações nos parâmetros de rede. Esse resultado poderá ser corroborado por métodos de analise química de maior acuracidade.

\section{CONCLUSÃO}

A rota de síntese foi eficiente na cristalização do material HT-HAp. O controle dos parâmetros temperatura, $\mathrm{pH}$, e a utilização de banho ultrassônico foram fatores determinantes na cristalização somente do material híbrido sem a presença de fases secundárias, entretanto, a fim de melhorar a cristalização do mesmo, modificações no tempo de envelhecimento do material poderão ser estudadas.

Os dados DRX indicaram a presença dos picos mais intensos tanto da HT quanto da HAp em concordância com a análise EDS que registrou os elementos $\mathrm{Ca}, \mathrm{P}, \mathrm{O}, \mathrm{Mg}$ e $\mathrm{Al}$ para o material estudado e razões $\mathrm{Ca} / \mathrm{P}=1,36$ e $\mathrm{Mg} / \mathrm{Al}=4,5$. O espectro FTIR confirmou a presença dos grupos inorgânicos $\mathrm{CO}_{3}{ }^{2-}, \mathrm{PO}_{4}{ }^{3-}, \mathrm{OH}^{-}$e $\mathrm{H}_{2} \mathrm{O}$, típicos dos minerais estudados.

O material híbrido apresenta potencial para futuros testes catalíticos pois acredita-se que as duas fases cristalizadas, HT e HAp, lhe conferem um caráter bifuncional. 


\section{9 a 22 de outubro de 2014 \\ Florianópolis/SC}

\section{REFERÊNCIAS}

AKAZAWA, T.; KOBAYASHI, M. Surface characteristics of hydroxyapatite controlling albumin adsorption behaviour. J. Mater. Sci. Lett. v. 15, p. 1319-1320, 1996.

BASTIANI, R. 2001. (Dissertação de Mestrado) - Universidade Federal do Rio de Janeiro, Brasil apud CONCEIÇÃO, L.; PERGHER, S. B. C.; MORO, C. C.; OLIVEIRA, L. C. A. Compósitos magnéticos baseados em hidrotalcitas para a remoção de contaminantes aniônicos em água. Quím. Nova. v.30, n.5, p. 1077-1081, 2007.

CARDOSO, L. P. Estudo da remoção de compostos orgânicos derivados da produção de poliéster presentes em efluentes industriais, por meio de sorção em hidróxidos duplos lamelares do sistema Mg-Al-CO3. 2002. 107f. (Dissertação de Mestrado) - Faculdade de Filosofia, Ciências e Letras de Ribeirão Preto, Universidade de São Paulo, Ribeirão Preto.

CAVANI, F.; TRIFFIRO, F.; VACCARI, A. Hydrotalcite-type anionic clays: preparation, properties and applications. Catal. Today, v. 11, p. 173-301, 1991.

CREPALDI, E. L.; VALIM, J. B. Hidróxidos Duplos Lamelares: Síntese, Estrutura, Propriedades e Aplicações. Quim. Nova, v. 21, n. 3, p. 300-311, 1998.

CUNHA, V.; FERREIRA, A. M. C.; CONSTANTINO, V. R. L.; TRONTO, J.; VALIM, J. B. Hidróxidos Duplos Lamelares: Nanopartículas Inorgânicas Para Armazenamento e Liberação de Espécies de Interesse Biológico e Terapêutico. Quim. Nova, v. 33, n. 1, p. 159-171, 2010.

EANES, E. D. Crystals growth of mineral phases in skeletal tissues. Prog. Crystal Growth Caracter. Mater. v. 3, p. 3-15, 1980.

FULMER, M. T.; MARTIN, R. I.; BROWN, P. W. Formation of Calcium deficient Hydroxyapatite at near-physiological temperature. J. Mater. Sci. - Mater. Med. v.3, p. 299-305, 1992.

GUASTALDI, A. C.; APARECIDA, A. H. Fosfatos de cálcio de interesse biológico: importância como biomateriais, propriedades e métodos de obtenção de recobrimentos. Quim. Nova. v. 33, n. 6, p. 1352-1358, 2010.

KAY, M. I.; YOUNG, R. A. Crystal Structure of Hydroxyapatite. Nature. v. 204, p.1050-1055, 1964 apud MAVROPOULOS, E. A hidroxiapatita como absorvedor de metais. 1999, 105f. (Dissertação de Mestrado) - Fundação Oswaldo Cruz, Escola Nacional de Saúde Pública, Rio de Janeiro.

MA, Q. Y.; TRAINA S. J.; LOGAN T. J.; E RYAN J. A. In situ lead immobilization by apatite. Environ. Sci. Technol. v. 27, p. 1803-1810, 1993.

REICHLE, W. T. Catalytic Reactions by Thermally Activated, Synthetic, Anionic Clay Minerals. $J$. Catal. v. 94, p. 547-557, 1985.

RIVERA, J. A.; FETTER, G.; BANOS, L.; GUZMAN, J.; BOSCH, P. New hydroxyapatitehydrotalcite composites I. synthesis. J Porous Mater, v. 16, p. 401-408, 2009.

TRAVE, A.; SELLONI, A.; GOURSOT, A.; TICHIT, D.; WEBER, J. First Principles Study of the Structure and Chemistry of Mg-Based Hydrotalcite-Like Anionic Clays. Journal Phys. Chemistry B. v. 106, p. 12291-12296, 2002. 